\title{
Atuação dos profissionais da equipe de enfermagem na farmacovigilância: revisão integrativa da literatura
}

\author{
Nursing team professionals' performance in pharmacovigilance: an \\ integrative literature review
}
Actuación profesional del equipo de enfermería en la farmacovigilancia: revisión integrativa de la literatura

Giovanna Gabrielly Custódio Macêdo¹, Mariana Albernaz Pinheiro de Carvalho²

\author{
${ }^{1}$ Graduanda em Enfermagem pela Universidade Federal de Campina Grande \\ Campina Grande - Paraíba. \\ ${ }^{2}$ Doutora em Enfermagem pela Universidade Federal da Paraíba. Docente da Universidade Federal \\ de Campina Grande . Campina Grande - Paraíba.
}

\section{RESUMO}

Este estudo teve por objetivo caracterizar a prática dos profissionais de enfermagem na farmacovigilância a partir da produção científica nacional e internacional dos últimos 10 anos. Revisão integrativa da literatura realizada no período de agosto a novembro de 2018 nas bases de dados Literatura Latino-Americana e do Caribe em Ciências da Saúde, Base de Dados de Enfermagem, Scientific Electronic Library Online e Medical Literature Analysis and Retrieval System Online, através de descritores preestabelecidos nos Descritores em Ciências da Saúde e cruzados por meio do operador booleano AND. Dos 57 artigos iniciais, 13 compuseram o corpus da pesquisa. Após análise temática e categorização, a atuação dos profissionais de enfermagem

Autor de Correspondência:

*Giovanna Gabrielly Custódio Macêdo. E-mail: cmacedogiovanna@hotmail.com 
contemplou as categorias Atuação assistencial da enfermagem na farmacovigilância e Atuação gerencial e educacional da enfermagem na farmacovigilância. Apesar de limitada e pouco concreta, a atuação da equipe de enfermagem se apresentou na linha de frente da vigilância do consumo de medicamentos.

Palavras-chave: Farmacovigilância. Profissionais de Enfermagem. Cuidados de Enfermagem.

\section{ABSTRACT}

This study aims to characterize the practice of nursing professionals in pharmacovigilance based on the national and international scientific production of the last 10 years. This is an integrative literature review conducted from August to November 2018 in the Latin American and Caribbean Health Sciences, Nursing Database, Scientific Electronic Library Online and Medical Literature Analysis and Retrieval System Online databases, through descriptors preestablished in the Descriptors in Health Sciences and crossed through the boolean operator AND. Of the 57 initial articles, 13 comprised the research corpus. Following thematic analysis and categorization, the performance of nursing professionals included the categories Nursing care practice in pharmacovigilance and Managerial and educational performance of nursing in pharmacovigilance. Although limited and not very concrete, the performance of the nursing team was at the forefront of drug use surveillance.

Keywords: Pharmacovigilance. Nurse Practitioners. Nursing Care.

\section{RESUMEN}

Caracterizar la práctica de los profesionales de enfermería en farmacovigilancia a partir de la producción científica nacional e internacional de los últimos 10 años. Revisión integral de la literatura realizada de agosto hasta noviembre de 2018 en las bases de datos en línea de las ciencias de la salud de América Latina y del Caribe, la enfermería, la biblioteca electrónica científica y el sistema de recuperación y análisis de literatura médica en línea, a través de descriptores preestablecidos en los Descriptores en Ciencias de la Salud y cruzados a través del operador booleano AND. De los 57 artículos iniciales, 13 comprendieron el corpus de investigación. Después del análisis temático y la categorización, el desempeño de los profesionales de enfermería incluyó las categorías Práctica de atención de enfermería en farmacovigilancia y Desempeño gerencial y educativo de enfermería en farmacovigilancia. Aunque limitado y poco concreto, el desempeño del equipo de enfermería estuvo a la vanguardia de la vigilancia del uso de drogas.

Palablas clave: Farmacovigilancia. Enfermeras Practicantes. Atención de Enfermería. Fuerza 


\section{INTRODUÇÃO}

A farmacovigilância se destina à detecção, avaliação, compreensão e prevenção de efeitos adversos ou de quaisquer outros problemas relacionados a medicamentos, além de incluir também os fitoterápicos, medicamentos complementares, hemoderivados, produtos biológicos, vacinas e dispositivos médicos, com o intuito de garantir que os benefícios inerentes à utilização desses produtos sejam maiores que os riscos ${ }^{1,2}$.

A prática da farmacovigilância na fase de póscomercialização de medicamentos assegura a construção de um perfil de segurança para o seu consumo $^{3}$. Dessa maneira, se lança como uma iniciativa específica no campo da segurança do paciente ao garantir a melhoria da qualidade de medicamentos ${ }^{4}$.

Estima-se que entre $2,9 \%$ e $16,6 \%$ dos pacientes hospitalizados vivenciam algum evento adverso durante a internação e àqueles relacionados ao uso de fármacos representam o segundo evento adverso mais frequente ${ }^{5}$. Entretanto, a dimensão da morbimortalidade por eventos adversos a medicamentos é limitada, dada a escassa produção científica que envolve a temática, o que pode contribuir com a cultura de não responsabilização profissional diante da segurança do paciente e o uso de medicamentos ${ }^{6}$.

Consoante a isso, apesar de os profissionais da equipe de enfermagem estarem na linha de frente de atividades que envolvem a farmacovigilância, sua prática profissional envolve inúmeros aspectos. Deste modo, cabe citar a atuação direta com o preparo e administração de medicamentos e a possibilidade de acompanhamento contínuo da situação de saúde do paciente frente ao uso de medicamentos e produtos para a saúde em geral ${ }^{7,8}$. Contudo, a participação da equipe de enfermagem nessa área é mínima.

Esse cenário é baseado em dois fatores centrais: frágil conhecimento sobre a farmacovigilância e a incipiente produção científica específica acerca da atuação da enfermagem em atividades que a envolvem. Nessa perspectiva, este estudo de revisão integrativa da literatura se baseou na seguinte questão norteadora: "Qual a atuação dos profissionais de enfermagem na farmacovigilância?”.

Tendo em vista a necessidade de robustecer a responsabilização da enfermagem nesse contexto, o presente estudo se propõe a caracterizar a prática dos profissionais de enfermagem na farmacovigilância a partir da produção científica nacional e internacional dos últimos 10 anos.

\section{MÉTODO}

Trata-se de uma pesquisa bibliográfica com caráter de revisão integrativa da literatura. Dessa maneira, os seguintes passos foram seguidos: identificação do tema e seleção da pesquisa, estabelecimento de critérios de inclusão e exclusão, seleção dos estudos, categorização dos estudos, análise e interpretação dos resultados 9 . A pesquisa foi conduzida pela questão norteadora: "Qual a atuação dos profissionais de enfermagem na farmacovigilância?".

A pesquisa foi composta por artigos obtidos através das bases de dados da Literatura LatinoAmericana e do Caribe em Ciências da Saúde (LILACS), Base de Dados de Enfermagem (BDEnf), Scientific Electronic Library Online (SciELO) e Medical Literature Analysis and Retrieval System Online (MEDLINE).

Consultou-se as bases de dados no período de agosto a novembro de 2018 através do cruzamento, por meio do operador booleano AND, dos descritores "Farmacovigilância" e "Profissionais de Enfermagem”, "Efeitos Colaterais e Reações Adversas Relacionados a Medicamentos" e "Cuidados de Enfermagem" nas bases BDEnf, LILACS e SciELO, 
e "Pharmacovigilance", "Drug-Related Side Effects and Adverse Reactions" e "Nursing Care" na base MEDLINE. As versões em português e inglês dos descritores estão preestabelecidas nos Descritores em Ciências da Saúde (DeCS). Ressalta-se que a diferenciação dos descritores e das modalidades em dupla ou em trio dos cruzamentos foi adotada para qualificar a busca a partir do perfil de cada base de dados.

Para a seleção da amostra inicial foram utilizados os seguintes critérios de inclusão: I) artigos indexados, II) idiomas português, inglês ou espanhol, III) publicados entre o período de 2009 e 2018, IV) publicação em revistas nacionais e internacionais, e V) artigos de pesquisa de campo relevantes para o tema, tendo como critério norteador a prática dos profissionais de enfermagem na farmacovigilância; e exclusão: I) artigos não disponíveis na íntegra, II) componentes de literatura cinzenta, como manuais e livros, III) artigos repetidos e IV) artigos nota prévia.

A discussão da pesquisa seguiu o procedimento de análise temática, constituída pelas etapas de pré-análise, categorização do material, disposição dos dados nas categorias e interpretação das informações ${ }^{10}$.

\section{RESULTADOS}

Conforme o processo de análise dos artigos obtidos, o quadro 1 a seguir demonstra o resultado do quantitativo de estudos a partir do cruzamento dos descritores em cada base de dados:

Quadro 1 - Distribuição dos resultados da pesquisa por cruzamento e base de dados

\begin{tabular}{|c|c|c|c|c|}
\hline Base de dados & \multirow{2}{*}{ BDEnf } & \multirow{2}{*}{ LILACS } & \multirow{2}{*}{ SciELO } & \multirow{2}{*}{ MEDLINE } \\
\hline Cruzamento & & & & \\
\hline $\begin{array}{l}\text { "Farmacovigilância" AND "Profissionais de } \\
\text { Enfermagem" }\end{array}$ & 2 & 11 & 4 & - \\
\hline $\begin{array}{l}\text { "Efeitos Colaterais e Reações Adversas Relacionados } \\
\text { a Medicamentos" AND "Cuidados de Enfermagem" }\end{array}$ & 11 & 5 & 0 & - \\
\hline $\begin{array}{l}\text { "Pharmacovigilance" AND "Drug-Related Side Effects } \\
\text { and Adverse Reactions" AND "Nursing Care" }\end{array}$ & - & - & - & 24 \\
\hline Total de artigos & 13 & 16 & 4 & 24 \\
\hline
\end{tabular}

Após leitura completa dos 57 artigos da amostra inicial e eliminação por meio dos critérios de inclusão e exclusão, o corpus de análise foi composto por13artigos, com obtenção de 3 na BDEnf, 4 LILACS, 0 SciELO e 6 PubMed.

A maior parte das exclusões ocorreu em virtude de
19 (43\%) artigos não abordarem a temática e 8 (18\%) possuírem tempo de publicação maior que o exigido para a inclusão. As demais exclusões se deram devido à indisponibilidade de acesso completo de 6 (14\%) artigos, 5 (11\%) repetições e por 6 (14\%) estudos serem teses, dissertações, monografias ou artigos nota prévia. 
De maneira a subsidiar a análise fidedigna dos artigos, caracterizou-se o corpus de análise conforme informações da autoria, publicação e pesquisa. O quadro 2 sistematiza as principais informações:

Quadro 2 - Sistematização do corpus de análise da pesquisa (continua)

\begin{tabular}{|c|c|c|c|c|}
\hline $\begin{array}{l}\text { Autores e ano } \\
\text { de publicação }\end{array}$ & $\begin{array}{l}\text { Título do } \\
\text { artigo }\end{array}$ & $\begin{array}{l}\text { Objetivo do } \\
\text { estudo }\end{array}$ & Metodologia & Desfecho \\
\hline $\begin{array}{l}\text { Bezerra ALQ, } \\
\text { Silva AEBC, } \\
\text { Branquinho } \\
\text { NCSS, Paranaguá } \\
\text { TTB, } 2009\end{array}$ & $\begin{array}{l}\text { Análise de queixas } \\
\text { técnicas e eventos } \\
\text { adversos notificados } \\
\text { em um hospital } \\
\text { sentinela }\end{array}$ & $\begin{array}{l}\text { Identificar os eventos } \\
\text { adversos ocorridos em } \\
\text { um hospital sentinela da } \\
\text { Região Centro-Oeste }\end{array}$ & $\begin{array}{l}\text { Pesquisa } \\
\text { retrospectiva } \\
\text { de aborgem } \\
\text { quantitativa }\end{array}$ & $\begin{array}{l}\text { Houveram eventos adversos } \\
\text { relacionados a medicamentos } \\
\text { e hemoderivados e queixas } \\
\text { técnicas de medicamentos e de } \\
\text { material médico-hospitalar }\end{array}$ \\
\hline $\begin{array}{l}\text { Varallo FR, } \\
\text { Planeta CS, } \\
\text { MastroianniI PC, } \\
2017\end{array}$ & $\begin{array}{l}\text { Effectiveness of } \\
\text { pharmacovigilance: } \\
\text { multifaceted } \\
\text { educational } \\
\text { intervention related } \\
\text { to the knowledge, } \\
\text { skills and attitudes } \\
\text { of multidisciplinary } \\
\text { hospital staff }\end{array}$ & $\begin{array}{l}\text { Avaliar o impacto } \\
\text { de uma intervenção } \\
\text { educativa multifacetada } \\
\text { em farmacovigilância } \\
\text { sobre os conhecimentos, } \\
\text { habilidades e atitudes } \\
\text { de profissionais de um } \\
\text { hospital }\end{array}$ & $\begin{array}{l}\text { Estudo } \\
\text { prospectivo, } \\
\text { aberto, não- } \\
\text { randomizado }\end{array}$ & $\begin{array}{l}\text { A intervenção melhorou o } \\
\text { preenchimento de formulários } \\
\text { e o conhecimento acerca da } \\
\text { farmacovigilância, além de } \\
\text { contribuir para detectar eventos } \\
\text { adversos graves induzidos por } \\
\text { drogas }\end{array}$ \\
\hline $\begin{array}{l}\text { Mendes D, Alves } \\
\text { C, Marques FB, } \\
2014\end{array}$ & $\begin{array}{l}\text { Nurses' spontaneous } \\
\text { reporting of adverse } \\
\text { drug reactions: expert } \\
\text { review of routine } \\
\text { reports }\end{array}$ & $\begin{array}{l}\text { Analisar as } \\
\text { reações adversas } \\
\text { a medicamentos } \\
\text { notificadas } \\
\text { espontaneamente } \\
\text { de acordo com a sua } \\
\text { descrição anterior, } \\
\text { gravidade, causalidade e } \\
\text { profissional relator }\end{array}$ & $\begin{array}{l}\text { Pesquisa } \\
\text { documental }\end{array}$ & $\begin{array}{l}\text { Os enfermeiros sabem } \\
\text { identificar reações adversas } \\
\text { graves, apesar de as relatarem } \\
\text { menos que outros profissionais. } \\
\text { É necessário que a categoria } \\
\text { assuma a responsabilidade de } \\
\text { notificar tais acontecimentos de } \\
\text { maneira rotineira }\end{array}$ \\
\hline $\begin{array}{l}\text { Silva RFA, } \\
\text { Barreiro Filho } \\
\text { RD, Santos M, } \\
\text { Nascimento MAL, } \\
2011\end{array}$ & $\begin{array}{l}\text { Estratégia } \\
\text { educacional como } \\
\text { contribuinte ao } \\
\text { gerenciamento de } \\
\text { risco hospitalar: } \\
\text { estudo quase } \\
\text { experimental }\end{array}$ & $\begin{array}{l}\text { Associar a estratégia } \\
\text { de sensibilização dos } \\
\text { profissionais através } \\
\text { de cartazes e folhetos } \\
\text { contendo informações } \\
\text { sobre os conceitos que } \\
\text { estão associados à Rede } \\
\text { Sentinela ao aumento de } \\
\text { notificações }\end{array}$ & $\begin{array}{l}\text { Estudo quase- } \\
\text { experimental }\end{array}$ & $\begin{array}{l}\text { Houve aumento nas notificações } \\
\text { relacionadas à tecnovigilância, } \\
\text { farmacovigilância e processo de } \\
\text { risco, exceto em hemovigilância. } \\
\text { O aprimoramento profissional } \\
\text { contribui para a qualidade do } \\
\text { cuidado prestado }\end{array}$ \\
\hline $\begin{array}{l}\text { Modesto ACF, } \\
\text { Ferreira TXAM, } \\
\text { Provin MP, } \\
\text { Amaral RG, Lima } \\
\text { DM, } 2016\end{array}$ & $\begin{array}{l}\text { Reações adversas } \\
\text { a medicamentos e } \\
\text { farmacovigilância: } \\
\text { conhecimentos } \\
\text { e condutas de } \\
\text { profissionais de } \\
\text { saúde de um hospital } \\
\text { da rede sentinela }\end{array}$ & $\begin{array}{l}\text { Identificar o } \\
\text { conhecimento e } \\
\text { as condutas dos } \\
\text { profissionais de saúde } \\
\text { de um hospital de } \\
\text { ensino, em relação } \\
\text { às reações adversas } \\
\text { a medicamentos e à } \\
\text { farmacovigilância e se } \\
\text { há associação com o seu } \\
\text { perfil profissiográfico }\end{array}$ & $\begin{array}{l}\text { Estudo } \\
\text { transversal }\end{array}$ & $\begin{array}{l}\text { Houve discrepâncias entre } \\
\text { a formação profissional e o } \\
\text { conhecimento e condutas } \\
\text { frente às reações adversas a } \\
\text { medicamentos }\end{array}$ \\
\hline
\end{tabular}




\begin{tabular}{|c|c|c|c|c|}
\hline $\begin{array}{l}\text { Autores e ano } \\
\text { de publicação }\end{array}$ & $\begin{array}{l}\text { Título do } \\
\text { artigo }\end{array}$ & $\begin{array}{l}\text { Objetivo do } \\
\text { estudo }\end{array}$ & Metodologia & Desfecho \\
\hline $\begin{array}{l}\text { Hammour KA, El- } \\
\text { Dahiyat F, Farha } \\
\text { RA, } 2017\end{array}$ & $\begin{array}{l}\text { Health care } \\
\text { professionals } \\
\text { knowledge and } \\
\text { perception of } \\
\text { pharmacovigilance } \\
\text { in a tertiary care } \\
\text { teaching hospital in } \\
\text { Amman, Jordan }\end{array}$ & $\begin{array}{l}\text { Avaliar a consciência, } \\
\text { conhecimento e } \\
\text { percepções de médicos } \\
\text { e enfermeiros em } \\
\text { relação ao seu papel } \\
\text { como repórteres de } \\
\text { reações adversas a } \\
\text { medicamentos na } \\
\text { Jordânia }\end{array}$ & $\begin{array}{l}\text { Estudo } \\
\text { transversal }\end{array}$ & $\begin{array}{l}\text { A maioria dos profissionais } \\
\text { de saúde não tinha } \\
\text { conhecimento do conceito } \\
\text { de farmacovigilância. Os } \\
\text { médicos mostraram um } \\
\text { melhor conhecimento geral em } \\
\text { comparação com os enfermeiros }\end{array}$ \\
\hline $\begin{array}{l}\text { Santos L, } \\
\text { Oliveira FR, } \\
\text { Martinbiancho } \\
\text { J, Jacoby T, } \\
\text { Mahmud SDP, Fin } \\
\text { MC et al, } 2012\end{array}$ & $\begin{array}{l}\text { Descrição das } \\
\text { notificações de } \\
\text { queixas técnicas } \\
\text { de medicamentos } \\
\text { recebidas pela } \\
\text { farmacovigilância do } \\
\text { Hospital de Clínicas } \\
\text { de Porto Alegre }\end{array}$ & $\begin{array}{l}\text { Descrever as } \\
\text { notificações } \\
\text { espontâneas de produtos } \\
\text { com suspeita de desvios } \\
\text { de qualidade, recebidas } \\
\text { pela farmacovigilância } \\
\text { do Hospital de Clínicas } \\
\text { de Porto Alegre no } \\
\text { período de } 2008 \text { a } 2012\end{array}$ & $\begin{array}{l}\text { Estudo } \\
\text { descritivo }\end{array}$ & $\begin{array}{l}\text { As notificações envolveram } \\
\text { problemas na embalagem } \\
\text { primária dos produtos e } \\
\text { na reconstituição dos pós } \\
\text { liofilizados, suspeita de falha } \\
\text { terapêutica, presença de corpo } \\
\text { estranho e reações adversas }\end{array}$ \\
\hline $\begin{array}{l}\text { Schutte T, van } \\
\text { Eekeren R, Richir } \\
\text { M, van Staveren J, } \\
\text { van Puijenbroek } \\
\text { E, Tichelaar J et } \\
\text { al, } 2017\end{array}$ & $\begin{array}{l}\text { The adverse drug } \\
\text { reaction reporting } \\
\text { assignment for } \\
\text { specialist oncology } \\
\text { nurses: a preliminary } \\
\text { evaluation of quality, } \\
\text { relevance and } \\
\text { educational value in } \\
\text { a prospective cohort } \\
\text { study }\end{array}$ & $\begin{array}{l}\text { Desenvolver e avaliar um } \\
\text { relatório de atribuições } \\
\text { de reações adversas } \\
\text { a medicamentos } \\
\text { para enfermeiros } \\
\text { especialistas em } \\
\text { oncologia }\end{array}$ & $\begin{array}{l}\text { Relato de } \\
\text { experiência }\end{array}$ & $\begin{array}{l}\text { O relatório ofereceu uma } \\
\text { abordagem prática e garantiu } \\
\text { maior atenção por parte dos } \\
\text { profissionais, além de mudar a } \\
\text { maneira como os profissionais } \\
\text { lidavam com reações adversas a } \\
\text { medicamentos }\end{array}$ \\
\hline $\begin{array}{l}\text { Angelis A, } \\
\text { Pancani L, Steca } \\
\text { P, Colaceci S, } \\
\text { Giusti A, Tibaldi } \\
\text { L et al, } 2017\end{array}$ & $\begin{array}{l}\text { Testing an explanatory } \\
\text { model of nurses' } \\
\text { intention to report } \\
\text { adverse drug reactions } \\
\text { in hospital settings }\end{array}$ & $\begin{array}{l}\text { Testar um modelo } \\
\text { explicativo da intenção } \\
\text { dos enfermeiros de } \\
\text { relatar reações adversas } \\
\text { a medicamentos em } \\
\text { ambientes hospitalares } \\
\text { com base na teoria } \\
\text { do comportamento } \\
\text { planejado }\end{array}$ & $\begin{array}{l}\text { Estudo } \\
\text { transversal }\end{array}$ & $\begin{array}{l}\text { A intenção dos enfermeiros } \\
\text { de relatar reações adversas } \\
\text { a medicamentos foi } \\
\text { significativamente prevista por } \\
\text { atitudes, normas subjetivas } \\
\text { e controle comportamental } \\
\text { percebido }\end{array}$ \\
\hline $\begin{array}{l}\text { Varallo FR, Passos } \\
\text { AC, Nadai TR, } \\
\text { Mastroianni PC, } \\
2018\end{array}$ & $\begin{array}{l}\text { Incidents reporting: } \\
\text { barriers and strategies } \\
\text { to promote safety } \\
\text { culture }\end{array}$ & $\begin{array}{l}\text { Identificar as barreiras } \\
\text { de subnotificação, os } \\
\text { fatores que promovem } \\
\text { a motivação dos } \\
\text { profissionais de } \\
\text { saúde para relatar e } \\
\text { as estratégias para } \\
\text { melhorar os relatos de } \\
\text { incidentes }\end{array}$ & $\begin{array}{l}\text { Técnica de } \\
\text { grupo de } \\
\text { conversação }\end{array}$ & $\begin{array}{l}\text { Os relatos foram dificultados } \\
\text { por aspectos como } \\
\text { complacência, medo de litígio, } \\
\text { ambição de publicar, ignorância } \\
\text { e falta de responsabilização. } \\
\text { O conhecimento é um fator } \\
\text { motivador, mas não é eficaz no } \\
\text { aumento de notificações }\end{array}$ \\
\hline
\end{tabular}




\begin{tabular}{|c|c|c|c|c|}
\hline $\begin{array}{l}\text { Autores e ano } \\
\text { de publicação }\end{array}$ & $\begin{array}{c}\text { Título do } \\
\text { artigo }\end{array}$ & $\begin{array}{l}\text { Objetivo do } \\
\text { estudo }\end{array}$ & Metodologia & Desfecho \\
\hline $\begin{array}{l}\text { Souza RFF, Silva } \\
\text { LD, } 2014\end{array}$ & $\begin{array}{l}\text { Estudo exploratório } \\
\text { das iniciativas } \\
\text { acerca da segurança } \\
\text { do paciente em } \\
\text { hospitais do Rio de } \\
\text { Janeiro }\end{array}$ & $\begin{array}{l}\text { Analisar, com base nas } \\
\text { informações obtidas } \\
\text { com os gerentes de } \\
\text { risco, as iniciativas } \\
\text { implementadas para } \\
\text { garantir a segurança do } \\
\text { paciente }\end{array}$ & $\begin{array}{l}\text { Estudo } \\
\text { exploratório }\end{array}$ & $\begin{array}{l}\text { Há realização de grande } \\
\text { parte das iniciativas atuais } \\
\text { para a segurança do paciente, } \\
\text { apesar de as relacionadas aos } \\
\text { medicamentos serem pouco } \\
\text { apontadas }\end{array}$ \\
\hline $\begin{array}{l}\text { Sarayani A, } \\
\text { Naderi-Behdani } \\
\text { F, Hadavand N, } \\
\text { Javadi M, Farsad } \\
\text { F, Hadjibabaie M } \\
\text { et al, } 2015\end{array}$ & $\begin{array}{l}\text { A 3-Armed } \\
\text { Randomized } \\
\text { Controlled Trial of } \\
\text { Nurses' Continuing } \\
\text { Education Meetings } \\
\text { on Adverse Drug } \\
\text { Reactions }\end{array}$ & $\begin{array}{l}\text { Comparar a taxa de } \\
\text { participação, satisfação } \\
\text { e o resultado da } \\
\text { aprendizagem de } \\
\text { reuniões baseadas em } \\
\text { oficinas versus reuniões } \\
\text { baseadas em palestras } \\
\text { para enfermeiros que } \\
\text { trabalham em um } \\
\text { hospital de referência } \\
\text { em tratamento } \\
\text { cardiovascular no Irã }\end{array}$ & $\begin{array}{l}\text { Ensaio } \\
\text { controlado } \\
\text { randomizado }\end{array}$ & $\begin{array}{l}\text { O grupo de oficinas mostrou } \\
\text { maior participação e satisfação } \\
\text { em comparação ao grupo de } \\
\text { palestras. No início do estudo } \\
\text { o nível de conhecimento foi } \\
\text { semelhante, após a reunião foi } \\
\text { maior no grupo de palestras e } \\
\text { ao final foi similar nos grupos } \\
\text { de oficinas e palestras e maior } \\
\text { no grupo controle }\end{array}$ \\
\hline $\begin{array}{l}\text { Pontes LPP, } \\
\text { Rocha TPO, Diniz } \\
\text { RP, Brito GMC, } \\
2017\end{array}$ & $\begin{array}{l}\text { Implantação da } \\
\text { gestão de risco } \\
\text { nos processos } \\
\text { relacionados a } \\
\text { medicamentos } \\
\text { utilizados em } \\
\text { unidade de terapia } \\
\text { intensiva }\end{array}$ & $\begin{array}{l}\text { Implantar a gestão de } \\
\text { risco nos processos } \\
\text { relacionados a } \\
\text { medicamentos } \\
\text { utilizados em terapia } \\
\text { intensiva na área de } \\
\text { farmacovigilância }\end{array}$ & $\begin{array}{l}\text { Relato de } \\
\text { experiência }\end{array}$ & $\begin{array}{l}\text { Foi possível a análise real das } \\
\text { principais necessidades na } \\
\text { área da farmacovigilância com } \\
\text { participação efetiva da gestão } \\
\text { de risco e enfoque na segurança } \\
\text { do paciente }\end{array}$ \\
\hline
\end{tabular}

As publicações que compuseram o corpus se concentraram no ano de 2017 (38\%) e 2014 (15\%), com 5 e 2 artigos, respectivamente, ao passo que houve uma publicação por ano em 2009, 2011, 2012, 2015, 2016 e 2018.

Aproximadamente $85 \%$ do corpus, que corresponde a 11 estudos, adotaram métodos de pesquisa de campo, enquanto 2 (15\%) artigos eram relatos de experiência. Um pouco mais da metade dos artigos (54\%) foram publicações internacionais, enquanto $6(46 \%)$ eram nacionais.

O quantitativo dos principais assuntos englobados nos estudos foi: 6 (46\%) artigos sobre eventos e reações adversas a medicamentos, 3 (23\%) sobre atuação das categorias profissionais na farmacovigilância, 2 (15\%) sobre gerenciamento de risco, 1 (8\%) sobre notificações e $1(8 \%)$ sobre segurança do paciente.

\section{DISCUSSÃO}

A partir da construção das duas categorias de análise, segue a apresentação dos estudos com base nos objetivos propostos. O quadro 3 apresenta as categorias formuladas, os artigos relacionados e as respectivas bases de dados: 
Quadro 3 - Categorização dos dados conforme análise temática

\begin{tabular}{|c|c|c|}
\hline Categoria & Título do artigo & Base de dados \\
\hline \multirow{10}{*}{$\begin{array}{l}\text { Atuação assistencial } \\
\text { da enfermagem na } \\
\text { farmacovigilância }\end{array}$} & $\begin{array}{l}\text { Análise de queixas técnicas e eventos adversos notificados em } \\
\text { um hospital sentinela }\end{array}$ & BDENF \\
\hline & $\begin{array}{l}\text { Effectiveness of pharmacovigilance: multifaceted educational } \\
\text { intervention related to the knowledge, skills and attitudes of } \\
\text { multidisciplinary hospital staff }\end{array}$ & MEDLINE \\
\hline & $\begin{array}{l}\text { Nurses' spontaneous reporting of adverse drug reactions: expert } \\
\text { review of routine reports }\end{array}$ & MEDLINE \\
\hline & $\begin{array}{l}\text { Estratégia educacional como contribuinte ao gerenciamento de } \\
\text { risco hospitalar: estudo quase experimental }\end{array}$ & BDENF \\
\hline & $\begin{array}{l}\text { Reações adversas a medicamentos e farmacovigilância: } \\
\text { conhecimentos e condutas de profissionais de saúde de um } \\
\text { hospital da rede sentinela }\end{array}$ & LILACS \\
\hline & $\begin{array}{l}\text { Health care professionals knowledge and perception of } \\
\text { pharmacovigilance in a tertiary care teaching hospital in Amman, } \\
\text { Jordan }\end{array}$ & MEDLINE \\
\hline & $\begin{array}{l}\text { Descrição das notificações de queixas técnicas de } \\
\text { medicamentos recebidas pela farmacovigilância do Hospital de } \\
\text { Clínicas de Porto Alegre }\end{array}$ & LILACS \\
\hline & $\begin{array}{l}\text { The adverse drug reaction reporting assignment for specialist } \\
\text { oncology nurses: a preliminary evaluation of quality, relevance and } \\
\text { educational value in a prospective cohort study }\end{array}$ & MEDLINE \\
\hline & $\begin{array}{l}\text { Testing an explanatory model of nurses' intention to report adverse } \\
\text { drug reactions in hospital settings }\end{array}$ & MEDLINE \\
\hline & Incidents reporting: barriers and strategies to promote safety culture & LILACS \\
\hline \multirow{5}{*}{$\begin{array}{l}\text { Atuação gerencial } \\
\text { e educacional da } \\
\text { enfermagem na } \\
\text { farmacovigilância }\end{array}$} & $\begin{array}{l}\text { Estudo exploratório das iniciativas acerca da segurança do } \\
\text { paciente em hospitais do Rio de Janeiro }\end{array}$ & BDENF \\
\hline & $\begin{array}{l}\text { Testing an explanatory model of nurses' intention to report adverse } \\
\text { drug reactions in hospital settings }\end{array}$ & MEDLINE \\
\hline & $\begin{array}{l}\text { Estratégia educacional como contribuinte ao gerenciamento de } \\
\text { risco hospitalar: estudo quase experimental }\end{array}$ & BDENF \\
\hline & $\begin{array}{l}\text { Implantação da gestão de risco nos processos relacionados a } \\
\text { medicamentos utilizados em unidade de terapia intensiva }\end{array}$ & LILACS \\
\hline & $\begin{array}{l}\text { Armed Randomized Controlled Trial of Nurses' Continuing } \\
\text { Education Meetings on Adverse Drug Reactions }\end{array}$ & MEDLINE \\
\hline
\end{tabular}




\section{Atuação assistencial da enfermagem na farmacovigilância}

Estudos nacionais mostram que os profissionais de enfermagem são os responsáveis pela maior parte das notificações de eventos adversos relacionados a medicamentos ${ }^{11,12}$, apesar de o quantitativo dessas notificações não refletir a real situação das irregularidades que envolvem o seu uso, visto que se sugere que eventos adversos acometam 10\% de todas as admissões hospitalares, enquanto apenas $1 \%$ dos incidentes são relatados ${ }^{12}$. Esse fato pode estar relacionado à condição voluntária que as notificações assumem ${ }^{11}$, apesar de a notificação espontânea ser considerada um bom método de identificação de eventos no intuito de mediar a criação de perfis de segurança para o consumo de medicamentos ${ }^{13}$.

Pesquisas internacionais, por sua vez, mostram que a quantidade de relatos de reações adversas feitos por enfermeiros é inferior à de médicos e farmacêuticos, ainda que sejam uma das principais causas de morbimortalidade relacionada ao processo de atenção à saúde ${ }^{13}$.

Apesar dessa atuação efetiva da equipe de enfermagem na realização de notificações no Brasil, um estudo realizado após a aplicação de estratégias educacionais demonstrou que as notificações que envolvem a farmacovigilância se detêm em sua maioria apenas a queixas técnicas ${ }^{14}$, que são irregularidades associadas ao afastamento dos parâmetros de qualidade exigidos para a comercialização de produtos para a saúde.

Esse cenário de subnotificação de reações adversas é justificado em alguns estudos pelo receio de punição que os profissionais possuem caso a ocorrência desses eventos seja associada a possíveis $\operatorname{erros}^{13,14}$. Nessa perspectiva, estima-se que somente $25 \%$ dos erros são notificados e apenas em situações que determinaram danos ao paciente, apesar de os enfermeiros serem os responsáveis por $86 \%$ das identificações de eventos adversos ocasionados por erros nas prescrições. Entretanto, quando os erros estão relacionados ao processo de administração, parte essencialmente ligada à equipe de enfermagem, apenas $2 \%$ são identificados ${ }^{14}$.

Além do receio de punição, falta de recursos educacionais e de investimento em programas de conscientização profissional, baixo conhecimento da equipe de enfermagem acerca das reações adversas a medicamentos, da importância das notificações espontâneas na geração de sinais de alerta para órgãos reguladores e de como realizá-las também são apontadas como fatores que contribuem para a baixa notificação ${ }^{12,13,15,16}$. É necessário considerar ainda que a falta de recursos humanos pode repercutir na disponibilização do tempo que seria voltado às notificações, de maneira a fazer com que os profissionais o destinem unicamente para as suas atividades assistenciais ${ }^{15}$.

Um estudo que analisou o conhecimento de diversos profissionais da saúde sobre aspectos gerais da farmacovigilância e reações adversas a medicamentos apontou que o baixo conhecimento de enfermeiros e técnicos em enfermagem, acerca dessa temática, pode estar relacionado a lacunas nos seus processos de formação acadêmica e desorganização das instituições de saúde frente às notificações e demais atividades que envolvem a farmacovigilância ${ }^{15}$.

De maneira geral, a prática dos profissionais de enfermagem nessas atividades envolve sua atuação direta com o preparo e administração de medicamentos, com o aprazamento de medicações e a necessidade de relacionar a finalidade terapêutica dos fármacos e a possibilidade de interações medicamentosas. Além disso, cabe destacar seu contato direto com o paciente e a viabilidade de reconhecimento em tempo hábil de reações adversas a medicamentos, fazendo com que esses profissionais sejam considerados um dos maiores vigilantes da qualidade de fármacos, a base das notificações de irregularidades e reações adversas e um dos principais influenciadores do sucesso de um sistema de farmacovigilância ${ }^{14,13,16-18}$. 
No entanto, a percepção de que a realização de notificações é uma prática inerente somente à equipe de enfermagem faz com que a cultura de não responsabilização diante da vigilância de medicamentos seja difundida entre os outros componentes da equipe de saúde. Nesse sentido, os profissionais de enfermagem apontam que a sobrecarga, a falta de tempo e a ausência de retorno acerca de encaminhamentos adotados a partir das notificações são as justificativas para a sua mínima participação em registros e construção de relatórios ${ }^{19,20,16}$

\section{Atuação gerencial e educacional da enfermagem na farmacovigilância}

Em âmbito gerencial, os profissionais de enfermagem atuam na coordenação de setores que lidam com a detecção de irregularidades que comprometem a qualidade e a segurança do uso de produtos para a saúde, como demonstrado em um estudo realizado no Rio de Janeiro, onde 93\% dos gerentes de risco envolvidos na pesquisa eram enfermeiros ${ }^{21}$.

Nessa pesquisa, a educação continuada foi a principal estratégia empregada para a disseminação de questões inerentes à segurança do paciente, apesar da implementação de sistemas de monitoramento de eventos adversos ser uma das ferramentas mais utilizadas atualmente ${ }^{21,19}$. Em conformidade a isso, o estudo demonstrou que as iniciativas menos implementadas pelos gerentes de riscos eram aquelas que envolviam o controle de medicamentos potencialmente perigosos e a prevenção de eventos adversos a medicamentos ${ }^{21}$.

Em contrapartida, a utilização de uma estratégia educacional, por meio da distribuição de cartazes e folhetos que abordavam conceitos sobre os hospitais sentinelas, gerência de risco, eventos adversos, tecnovigilância, farmacovigilância, hemovigilância, processo de risco e notificação, demonstrou associação com o aumento do número de notificações ${ }^{14}$. Intervenções educacionais baseadas em oficinas, palestras e distribuição de materiais educativos também se mostraram efetivas no aperfeiçoamento de profissionais de enfermagem e de saúde em geral sobre aspectos relacionados à farmacovigilância e às reações adversas a medicamentos 22,12 .

Além de implantar métodos de sensibilização frente à coordenação de setores de vigilância, os profissionais de enfermagem que atuam no cuidado direto têm a possibilidade de participar da implementação de medidas que evitam problemas relacionados ao uso de medicamentos, como foi realizado em um estudo a partir das estratégias gerenciais de Plano Estratégico Situacional, Plano Operativo e Diagrama de Ishikawa ${ }^{23}$.

A limitação deste estudo envolveu o reduzido quantitativo de artigos encontrados, de modo a limitar o aprofundamento da temática. No entanto, suscita a necessidade de que pesquisas subsequentes abordem a atuação específica do profissional da equipe de enfermagem nesse contexto, ponderandose sua ampla atividade na área.

Nessa perspectiva, sugere-se a realização de amplas pesquisas de campo de caráter transversal e de intervenção para explicitar o cenário do envolvimento dos profissionais da equipe de enfermagem em medidas de vigilância de produtos para a saúde e de segurança do paciente, no intuito de qualificar a assistência dessa categoria e garantir boas práticas de cuidado à saúde.

Os resultados obtidos contribuem para a consolidação da atuação dessa categoria frente às irregularidades que envolvem a farmacoterapia e, consequentemente, para a efetivação de medidas de segurança do paciente diante do uso de produtos para a saúde de maneira geral, além de fomentar a necessidade de implementação institucional de práticas de educação permanente que assegurem a qualidade do consumo de medicamentos. 


\section{CONCLUSÕES}

Os resultados deste estudo apontam a atuação dos profissionais da equipe de enfermagem em áreas de assistência direta e indireta ao paciente, destacando-se prioritariamente o seu envolvimento com a percepção de irregularidades e a realização de notificações, seguido das ações relacionadas à gerência de setores de vigilância e a aplicabilidade e implementação de estratégias administrativas e educacionais.

Apesar da notável importância desses profissionais no contexto da farmacovigilância e da segurança do paciente diante do consumo de medicamentos, sua participação é pouco concreta em ambas as áreas. Os estudos demonstram que a corresponsabilização da enfermagem nesses cenários não é uma atividade desempenhada de maneira difundida e padronizada, contribuindo para a fragmentação da assistência prestada, insegurança no consumo de medicamentos em sua fase de pós-comercialização e restrita atuação profissional.

O reduzido quantitativo de estudos encontrados que contemplem a atuação específica da enfermagem nessa área demonstra o déficit referente ao envolvimento da categoria em atividades de farmacovigilância, e contribui para a manutenção desse cenário.

Faz-se necessário que setores de instituições hospitalares destinados à vigilância de produtos para a saúde invistam na criação de estratégias educativas de caráter permanente que os insiram em atividades de aperfeiçoamento teórico, com o intuito de sensibilizar os profissionais acerca da necessidade da sua atuação contínua e de qualidade nessas atividades.

\section{Agradecimentos}

Ao Conselho Nacional de Desenvolvimento Científico e Tecnológico.

\section{REFERÊNCIAS}

1. Organização Pan-Americana da Saúde. Boas práticas de farmacovigilância para as Américas. Washington DC: OPAS; 2011 [acesso em 18 nov 2018]. Disponível em: http://iris.paho.org/xmlui/ bitstream/handle/123456789/28455/9789275731604_spa. pdf? sequence $=1 \&$ is Allowed $=y$

2. Agência Nacional de Vigilância Sanitária (Brasil). Farmacovigilância. 2016 [acesso em 18 nov 2018]. Disponível em: http://portal.anvisa.gov.br/farmacovigilancia

3. Agência Nacional de Vigilância Sanitária (Brasil). Reações Adversas a Medicamentos. 2016 [acesso em 18 nov 2018]. Disponível em: http://portal.anvisa.gov.br/ resultado-de-busca?p_p_id=101\&p_p_lifecycle=0\&p_p_ state $=$ maximized \& _ $_{\text {_ }}$ mode $=$ view $\& p_{-} p_{-} c_{-} l_{-}$ id $=$ column-1\&p_p_col_count=1\&_101_struts_ action=\%2Fasset_publisher\%2Fview_content\&_101_ assetEntryId=2894491\&_101_type=document

4. Ministério da Saúde (Brasil). Documento de Referência para o Programa Nacional de Segurança do Paciente. Brasília: Ministério da Saúde; 2014 [acesso em 18 nov 2018]. Disponível em:

http://bvsms.saude.gov.br/bvs/publicacoes/documento_ referencia_programa_nacional_seguranca.pdf

5. Lorenzini E, Santi JAR, Bao ACP. Segurança do paciente: análise dos incidentes notificados em um hospital do sul do Brasil. Rev Gaúch Enferm. 2014 [acesso em 26 nov 2018]; 35(2):121-7. DOI: 10.1590/1983-1447.2014.02.44370

6. Sousa LAO, Fonteles MMF, Monteiro MP, Mengue SS, Bertoldi AD, Pizzol TSD et al. Prevalência e características dos eventos adversos a medicamentos no Brasil. Cad Saúde Pública. 2018 [acesso em 26 nov 2018]; 34(4):e00040017. DOI: 10.1590/0102-311X00040017

7. Pezato TPJ, Cesaretti MLR. Farmacovigilância hospitalar: treinamento de profissionais na potencialização de suas ações. Rev Fac Ciênc Med Sorocaba [Internet] 2015 [acesso em 18 nov 2018]; 17(3):135-139. Disponível em: https:// revistas.pucsp.br/index.php/RFCMS/article/viewFile/23518/ pdf

8. Duarte ML, Batista LM, Albuquerque PMS. Notificações de farmacovigilância em um hospital oncológico sentinela da Paraíba. Revista Brasileira de Farmacologia Hospitalar e Serviços de Saúde [Internet] 2014 [acesso em 18 nov 2018]; 5(1):7-11. Disponível em: http://www.sbrafh.org.br/rbfhss/ 
public/artigos/2014050101000470BR.pdf

9. Botelho LLR, Cunha CCA, Macedo M. O método da revisão integrativa nos estudos organizacionais. Gestão e Sociedade [Internet] 2011 [acesso em 18 nov 2018]; 5(11):121136. Disponível em: https://www.gestaoesociedade.org/ gestaoesociedade/article/view/1220/906

10. Bardin L. Análise de conteúdo. São Paulo: Edições 70; 2011

11. Bezerra ALQ, Silva AEBC, Branquinho NCSS, Paranaguá TTB. Análise de queixas técnicas e eventos adversos notificados em um hospital sentinela. Rev enferm UERJ [Internet] 2009 [acesso em 01 dez 2018]; 17(4):467-72. Disponível em: http://www.facenf.uerj.br/v17n4/v17n4a02. pdf

12. Varallo FR, Planeta CS, Mastroianni PC. Effectiveness of pharmacovigilance: multifaceted educational intervention related to the knowledge, skills and attitudes of multidisciplinary hospital staff. Clinics. 2017 [acesso em 01 dez 2018]; 72(1):51-57. DOI: 10.6061/clinics/2017(01)09

13. Mendes D, Alves C, Marques FB. Nurses' spontaneous reporting of adverse drug reactions: expert review of routine reports. J Nurs Manag. 2014 [acesso em $01 \mathrm{dez}$ 2018]; 22(3):322-330. DOI: 10.1111/jonm.12003

14. Silva RFA, Barreiro Filho RD, Santos M, Nascimento MAL. Estratégia educacional como contribuinte ao gerenciamento de risco hospitalar: estudo quaseexperimental. Online Braz J Nurs [Internet] 2011 [acesso em 01 dez 2018]; 10(1):1-11. Disponível em: http://www.objnursing.uff.br/index.php/nursing/rt/ printerFriendly/3221/794

15. Modesto ACF, Ferreira TXAM, Provin MP, Amaral RG, Lima DM. Reações adversas a medicamentos e farmacovigilância: conhecimentos e condutas de profissionais de saúde de um hospital da rede sentinela. Rev Bras Educ Méd. 2016 [acesso em 01 dez 2018]; 40(3):401410. DOI: 10.1590/1981-52712015v40n3e01502015

16. Hammour KA, El-Dahiyat F, Farha RA. Health care professionals knowledge and perception of pharmacovigilance in a tertiary care teaching hospital in Amman, Jordan. J Eval Clin Pract. 2017 [acesso em 01 dez 2018]; 23(3):608-613. DOI: 10.1111 / jep.12683
17. Santos L, Oliveira FR, Martinbiancho J, Jacoby T, Mahmud SDP, Fin MC et al. Descrição das notificações de queixas técnicas de medicamentos recebidas pela farmacovigilância do Hospital de Clínicas de Porto Alegre. Rev HCPA \& Fac Med Univ Fed Rio Gd do Sul [Internet] 2012 [acesso em 01 dez 2018]; 32(4):490-495. Disponível em: https://seer.ufrgs.br/hcpa/article/view/31885/23937

18. Schutte T, van Eekeren R, Richir M, van Staveren J, van Puijenbroek E, Tichelaar J et al. The adverse drug reaction reporting assignment for specialist oncology nurses: a preliminary evaluation of quality, relevance and educational value in a prospective cohort study. NaunynSchmiedeberg's Arch Pharmacol. 2017 [acesso em $01 \mathrm{dez}$ 2018]; 391(1):17-26. DOI: 10.1007 / s00210-017-1430-z

19. Angelis A, Pancani L, Steca P, Colaceci S, Giusti A, Tibaldi L et al. Testing an explanatory model of nurses' intention to report adverse drug reactions in hospital settings. J Nurs Manag. 2017 [acesso em 01 dez 2018; 25(4):307-317. DOI: 10.1111 / jonm. 12467

20. Varallo FR, Passos AC, Nadai TR, Mastroianni PC. Incidents reporting: barriers and strategies to promote safety culture. Rev Esc Enferm USP. 2018 [acesso em $01 \mathrm{dez}$ 2018]; 52:e03346. DOI: 10.1590/S1980-220X2017026403346

21. Souza RFF, Silva LD. Estudo exploratório das iniciativas acerca da segurança do paciente em hospitais do Rio de Janeiro. Rev enferm UERJ [Internet] 2014 [acesso em $01 \mathrm{dez}$ 2018]; 22(1):22-8. Disponível em: https://www.e-publicacoes. uerj.br/index.php/enfermagemuerj/article/view/11399/8972

22. Sarayani A, Naderi-Behdani F, Hadavand N, Javadi M, Farsad F, Hadjibabaie M et al. A 3-Armed Randomized Controlled Trial of Nurses' Continuing Education Meetings on Adverse Drug Reactions. J Contin Educ Health Prof. 2015 [acesso em 01 dez 2018]; 35(2):123-130. DOI: 10.1002 / chp. 21276

23. Pontes LPP, Rocha TPO, Diniz RP, Brito GMC. Implantação da gestão de risco nos processos relacionados a medicamentos utilizados em unidade terapia intensiva. Rev Baiana de Saúde Pública [Internet] 2017 [acesso em 01 dez 2018]; 41(3):804-813. Disponível em: http://rbsp.sesab. ba.gov.br/index.php/rbsp/article/download/2326/2322/ 